\title{
Conserved amino acid residues found in a predicted cytosolic domain of the lipopolysaccharide biosynthetic protein WecA are implicated in the recognition of UDP- $N$ - acetylglucosamine
}

\author{
Amal O. Amer ${ }^{1}$ and Miguel A. Valvano ${ }^{1,2}$ \\ Author for correspondence: Miguel A. Valvano. Tel: +1 519661 3996. Fax: +1 5196613499. \\ e-mail: mvalvano@uwo.ca
}

Departments of Microbiology and Immunology ${ }^{1}$ and Medicine, 2 The University of Western Ontario, London, Ontario, Canada N6A 5 C1

\begin{abstract}
WecA, an integral membrane protein that belongs to a family of polyisoprenyl phosphate $\boldsymbol{N}$-acetylhexosamine-1-phosphate transferases, is required for the biosynthesis of 0 -specific LPS and enterobacterial common antigen in Escherichia coli and other enteric bacteria. WecA functions as an UDP-Nacetylglucosamine (GICNAC) : undecaprenyl-phosphate GIcNAC-1-phosphate transferase. A conserved short sequence motif (His-Ile-His-His; HIHH) and a conserved arginine were identified in WecA at positions 279-282 and 265, respectively. This region is located within a predicted cytosolic segment common to all bacterial homologues of WecA. Both $\mathrm{HIHH}_{279-282}$ and the $\mathrm{Arg}_{265}$ are reminiscent of the HIGH motif (His-lle-Gly-His) and a nearby upstream lysine, which contribute to the three-dimensional architecture of the nucleotide-binding site among various enzymes displaying nucleotidyltransferase activity. Thus, it was hypothesized that these residues may play a role in the interaction of WecA with UDP-GICNAc. Replacement of the entire HIHH motif by site-directed mutagenesis produced a protein that, when expressed in the $E$. coli wecA mutant MV501, did not complement the synthesis of 07 LPS. Membrane extracts containing the mutated protein failed to transfer UDP-GICNAC into a lipid-rich fraction and to bind the UDP-GICNAC analogue tunicamycin. Similar results were obtained by individually replacing the first histidine $\left(\mathrm{H}_{279}\right)$ of the $\mathrm{HIHH}$ motif as well as the $\mathrm{Arg}_{265}$ residue. The functional importance of these residues is underscored by the high level of conservation of $\mathrm{H}_{279}$ and $\mathrm{Arg}_{265}$ among bacterial WecA homologues that utilize several different UDP-N-acetylhexosamine substrates.
\end{abstract}

Keywords: undecaprenol, O antigen biosynthesis, membrane protein, transferase, tunicamycin

\section{INTRODUCTION}

WecA is an UDP-N-acetylglucosamine (GlcNAc): undecaprenyl-phosphate (Und-P) GlcNAc-1-phosphate transferase that belongs to a large family of eukaryotic and prokaryotic polyisoprenyl-phosphate $\mathrm{N}$ acetylhexosamine-1-phosphate transferases (Anderson et al., 2000; Dal Nogare \& Lehrman, 1988; Lehrman, 1994; Rick \& Silver, 1996). The members of this family

Abbreviations: GlcNAc, $N$-acetylglucosamine; Und-P, undecaprenyl phosphate. catalyse the formation of a phosphodiester bond between a membrane-associated polyprenol phosphate molecule and $\mathrm{N}$-acetylhexosamine 1-phosphate, which is usually donated by a soluble UDP- $N$-acetylhexosamine precursor. WecA participates in the biosynthesis of O antigen LPS in many enteric bacteria and it is also involved in the biosynthesis of enterobacterial common antigen (Rick \& Silver, 1996). Although WecA has not been purified to homogeneity there is sufficient biochemical and genetic information to support its role in the transfer of GlcNAc 1-phosphate from UDPGlcNAc onto Und-P to form Und-P-P-GlcNAc (Rick \& 
Table 1. Bacterial strains and plasmids used in this study

\begin{tabular}{|c|c|c|}
\hline $\begin{array}{l}\text { Strain or } \\
\text { plasmid }\end{array}$ & Relevant properties & Source or reference \\
\hline \multicolumn{3}{|l|}{$\begin{array}{l}\text { E. coli } \\
\text { strains }\end{array}$} \\
\hline CLM20 & lacZ $\operatorname{trp} \Delta(s b c B-r f b)$ upp rel $r p s L$ rec $A \Delta(w e c A C)$ & Feldman et al. (1999) \\
\hline $\mathrm{DH} 5 \alpha$ & $\mathrm{F}^{-} \phi 80$ lacZM15 endA recA hsdR $\left(\mathrm{r}_{\mathrm{K}}^{-} \mathrm{m}_{\mathrm{K}}^{-}\right)$supE thi gyrA relA $\Delta(\operatorname{lac} Z Y A-\arg F) U 169$ & Laboratory stock \\
\hline JT4000 & Alac rpsL relA $A^{+}$araD139 flbB 4 lon510 & $\begin{array}{l}\text { S. Gottesman, NIH, Bethesda, } \\
\text { USA }\end{array}$ \\
\hline MV501 & VW187; wecA::Tn10, Tet $^{\mathrm{R}}$ & Alexander \& Valvano (1994) \\
\hline XL-1 Blue & $\mathrm{F}^{-}$recA endA gyrA thi-1 hsdR supE relA lac $\left[\mathrm{F}^{\prime}\right.$ proAB $\operatorname{lacl}^{\mathrm{q}} \mathrm{Z} \Delta M 15$ Tn10 $\left(\right.$ Tet $\left.\left.^{\mathrm{R}}\right)\right]$ & Stratagene \\
\hline \multicolumn{3}{|l|}{ Plasmids } \\
\hline pAA9 & $1 \cdot 2 \mathrm{~kb}$ fragment containing $w e c A_{\mathrm{E} 320}$ tagged with FLAG sequence & This study \\
\hline pAA10 & $\begin{array}{l}\text { pAA12 containing } w e c A_{\text {MalFTM }} \text { with the } 54 \text { bp fragment encoding } w e c A \text { TMXI } \\
\text { swapped with the } 90 \mathrm{bp} \text { fragment encoding the last transmembrane domain of } \\
\text { MalF }\end{array}$ & This study \\
\hline pAA12 & $\begin{array}{l}1.4 \mathrm{~kb} \text { fragment containing tagged } w e c A \text { cloned in pBluescript II } \mathrm{KS}+; w e c A^{+} \text {, } \\
\mathrm{Amp}^{\mathrm{R}}\end{array}$ & Amer \& Valvano (2000) \\
\hline pAA26 & $\begin{array}{l}1 \cdot 2 \mathrm{~kb} \text { EcoRI-PvuII fragment from pAA14 (Amer \& Valvano, 2000) containing } \\
w e c A_{\mathrm{FLAG}} \text { ligated into EcoRI and SmaI sites of pBAD24, Amp }\end{array}$ & This study \\
\hline pAA33 & pAA26 containing $w e c A_{\mathrm{HIHH} / \mathrm{GGGG}}$ & This study \\
\hline pAA51 & pAA26 containing $w e c A_{\mathrm{H} 279 \mathrm{~s}}$ & This study \\
\hline pAA52 & pAA26 containing $w e c A_{\mathrm{R} 265 \mathrm{~K}}$ & This study \\
\hline pBAD24 & Cloning vector, $\mathrm{Amp}^{\mathrm{R}}$ & Guzman et al. (1995) \\
\hline pMF21 & $1.5 \mathrm{~kb}$ PCR amplicon containing $w z x_{\mathrm{EcO}}$ cloned into pEXT21, $\mathrm{Spc}^{\mathrm{R}}$ & Feldman et al. (1999) \\
\hline
\end{tabular}

Silver, 1996). The product of this reaction serves as an acceptor for the addition of subsequent sugars to complete the biosynthesis of $\mathrm{O}$ antigen or enterobacterial common antigen repeating subunits. In the case of the $\mathrm{O} 7$ polysaccharide, a model system used in our laboratory, the repeating subunit consists of a backbone of GlcNAc, galactose, mannose and $\mathrm{N}$ acetylviosamine, and a side chain of rhamnose (L'Vov et al., 1984; Marolda et al., 1999). Once complete, the O7 subunit is translocated to the periplasmic surface of the inner membrane by a process that requires the Wzx protein (Feldman et al., 1999). O7 subunits are polymerized and subsequently ligated to preformed lipid A core oligosaccharide to form the complete O7 LPS molecule (Feldman et al., 1999; Marolda et al., 1999).

Comparisons of amino acid sequences among the members of the polyisoprenyl-phosphate $\mathrm{N}$-acetylhexosamine-1-phosphate transferase family reveal an alternate clustering of hydrophobic and hydrophilic domains, suggesting the presence of multiple transmembrane regions. The topology of two members of this family, the bacterial protein MraY that is involved in peptidoglycan synthesis (Bouhss et al., 1999) and the eukaryotic GPT that is involved in protein glycosylation (Dan et al., 1996), has been characterized experimentally. Bacterial and eukaryotic proteins share discrete regions of conserved amino acid sequence, especially those located in hydrophilic segments of the protein that are exposed to the cytosolic face of the plasma membrane or the membrane of the endoplasmic reticulum (Bouhss et al., 1999; Dal Nogare \& Lehrman, 1988).
Bacterial homologues also carry a large cytosolic loop that shares very little similarity with the eukaryotic members of the family (Bouhss et al., 1999; Dal Nogare \& Lehrman, 1988). It has recently been proposed that this large cytosolic region, ranging from approximately 32 to 43 aa in length, may be important for the recognition of the nucleotide-sugar substrates and may also determine the specificity of such an interaction (Anderson et al., 2000). In a previous study, we have constructed a functional WecA derivative carrying a FLAG epitope tag, which was fused to the C-terminal end of the protein (Amer \& Valvano, 2000). Using the $\mathrm{WecA}_{\mathrm{FLAG}}$ construct, we have determined the role of aspartic acid residues located in two distinct regions within WecA, both of which are predicted to correspond to cytosolic-exposed segments of the protein (A. O. Amer \& M. A. Valvano, unpublished). These residues may contribute to the formation of the phosphodiester bond and the interaction with divalent cations that is essential for catalytic activity (A. O. Amer \& M. A. Valvano, unpublished). In this study, we have modelled the topology of WecA taking into account the available experimental data from the topological analysis of MraY (Bouhss et al., 1999), and examined in detail the role of a conserved motif within the predicted large cytosolic loop.

\section{METHODS}

Strains and plasmids. The characteristics of the Escherichia coli strains and plasmids used in this study are described in Table 1. E. coli strains DH5 $\alpha$ and XL-1 Blue were used for all 
Table 2. Oligonucleotide primers used in this work

The nucleotide sequence encoding the last transmembrane domain in MalF is underlined. Mutagenic oligonucleotides are bold and underlined.

\begin{tabular}{|c|c|c|}
\hline WecA mutant & $\begin{array}{c}\text { Primer } \\
\text { no. }\end{array}$ & Sequence $\left(5^{\prime}-3^{\prime}\right)$ \\
\hline \multirow[t]{2}{*}{$\mathrm{WecA}_{320}$} & 62 & TCGATGCAATGGAAT \\
\hline & 191 & CACTCCGGGACAAAATG \\
\hline \multirow[t]{6}{*}{ WecA $_{\text {MalFтм }}$} & 185 & GGCGTGCTGGCAGAATATTCTCATTTTGTCCCGGAG \\
\hline & & TTTGAAGGCGGCGGGGGTCAGGACTTCGGTCTGGC \\
\hline & & $\underline{\text { GGCAGCAATTGCC }}$ \\
\hline & 186 & GCGCTTAATAAAGCGAGCAACTTTCCAGGCACGCTT \\
\hline & & CAGGTTGACTATCGCCAGCGCACCCACCAGCAGGAA \\
\hline & & $\underline{\text { GATCAGCGT }}$ \\
\hline \multirow{4}{*}{$\mathrm{WecA}_{\mathrm{HIHH} / \mathrm{GGGG}}$} & 165 & CTCGAGTCACTTGTCGTC \\
\hline & 170 & ATTACGAATTCGGTGAATTTACTGACAGTG \\
\hline & 237 & GGGGGATTGATCATGCGTGCCGGG \\
\hline & 238 & $\underline{\text { ACCTCCCTGACGGTCAGGAGAGAA }}$ \\
\hline \multirow[t]{2}{*}{$\mathrm{WecA}_{\mathrm{H} 279 \mathrm{~S}}$} & 544 & CCTGACCGTCAGTCTATTCACCATTTGATCATGCGTGCC \\
\hline & 545 & GGCACGCATGATCAAATGGTGAATAGACTGACGGTCAGG \\
\hline \multirow[t]{2}{*}{$\mathrm{WecA}_{\mathrm{R} 265 \mathrm{~K}}$} & 546 & $\begin{array}{l}\text { GGATATGGTGGCGATTATGTACCGTAAACTGCGTAAAGGC } \\
\text { ATGAGC }\end{array}$ \\
\hline & 547 & $\begin{array}{l}\text { GCTCATGCCTTTACGCAGTTTACGGTACATAATCGCCACCA } \\
\text { TATCC }\end{array}$ \\
\hline
\end{tabular}

plasmid manipulations, and E. coli JT4000 was used for the expression of WecA $\mathrm{WalFTm}_{\text {and }}$ WecA $\mathrm{E} 320$. Competent cells were prepared for transformation by either the calcium chloride method or electroporation as described elsewhere (Cohen et al., 1972; Dower et al., 1988). Bacteria were cultured in Luria-Bertani (LB) medium supplemented with $100 \mu \mathrm{g}$ ampicillin $\mathrm{ml}^{-1}$ or $20 \mu \mathrm{g}$ tetracycline $\mathrm{ml}^{-1}$ and $0.02 \%$ $(\mathrm{w} / \mathrm{v})$ arabinose as appropriate. All reagents were from Sigma, unless otherwise indicated.

General methods for plasmid constructions. Mutagenic oligonucleotides are listed in Table 2. PCR reactions were carried out with a Hybaid Ommigene thermocycler (Interscience) and conducted with PwoI DNA polymerase (Roche Diagnostics) unless otherwise indicated. DNA fragments were purified from $0.7 \%$ agarose gels with the QIAquick gel extraction kit (Qiagen) and ligated with T4 DNA ligase (Roche Diagnostics) to the corresponding plasmid vectors. All constructs made in this study were confirmed by sequencing the entire wecA gene with an automated sequencer at the DNA Sequencing Facility, Robarts Research Institute, London, Ontario. Plasmid DNA for sequencing was prepared with the High Purity Plasmid Preparation Kit (Qiagen).

Construction of the wec $A_{\mathrm{E} 320}$ gene encoding a FLAG-tagged C-terminal deletion of WecA. pAA26 DNA template encoding the parental $w e c A_{\mathrm{FLAG}}$, the sense primer 62 , incorporating an EcoRI site, and the antisense primer 191, encoding the Cterminal part of the wecA gene up to the glutamic acid residue at position $320\left(\mathrm{E}_{320}\right)$ were used in a PCR reaction. The DNA product was digested with EcoRI and SmaI, and ligated into the pAA8 vector (Amer \& Valvano, 2000), which had also been cleaved with EcoRI and $S m a \mathrm{I}$. This construct, designated pAA9, resulted in a wecA gene lacking the coding region for the last transmembrane domain and the C-terminal tail of the protein $\left(w e c A_{\mathrm{E} 320}\right)$. Primer 191 was designed in such a way that the FLAG epitope tag in pAA8 was fused in-frame with wec $A_{\mathrm{E} 320}$.

Construction of the wec $A_{\text {MalFTM }}$ gene encoding a FLAG-tagged WecA protein with a swapped last transmembrane domain. pAA12 (Amer \& Valvano, 2000) was first linearized by digestion with $\mathrm{NheI}$, which cuts in the middle of the sequence encoding the last transmembrane domain of WecA. This fragment was used as a template for a PCR reaction with primers 185 (sense) and 186 (antisense). Primer 185 was designed to encode part of the fifth external loop region of the wecA gene followed by the sequence encoding the $\mathrm{N}$-terminal half of the last transmembrane domain of the MalF protein (Boyd et al., 1987). Primer 186 encoded part of the wecA gene C-terminal tail and the C-terminal half of the MalF last transmembrane domain with the introduction of a HincII site to facilitate the identification of the new construct. The PCR product was purified, self-ligated and transformed into E. coli $\mathrm{DH} 5 \alpha$, resulting in the isolation of pAA10. This plasmid contained a wecA gene encoding a WecA protein in which the last transmembrane domain was replaced by the last transmembrane segment of MalF (WecA $\mathrm{MalFTM}_{\text {M }}$ ).

Oligonucleotide-directed mutagenesis of wecA. The replacement of the $\mathrm{HHIH}_{279-282}$ motif by four glycines was conducted in two steps. Primer 165, which anneals to the FLAG sequence, was used in reactions amplifying the C-terminal part of wecA, and primer 170, which contains an EcoRI cleavage site, was used in reactions amplifying the $\mathrm{N}$-terminal portion of wecA. A $0.26 \mathrm{~kb}$ C-terminal fragment of wecA was first amplified by PCR using antisense primer 165 and sense primer 237, which contains the codons for two glycine residues to substitute $\mathrm{His}_{281}$ and $\mathrm{His}_{282}$. This fragment was ligated into the $\mathrm{SmaI}$ site of pBAD24. Then the N-terminal part of wecA was amplified using sense primer 170 and antisense primer 238, which contains the codons for the other two glycine residues 
replacing $\mathrm{His}_{279}$ and $\mathrm{Ile}_{280}$ of the $\mathrm{HIHH}$ motif. The amplified fragment was ligated into the previous construct after digesting it with EcoRI and SmaI, producing pAA33. This strategy resulted in the reconstruction of a full-length wecA gene tagged with the FLAG sequence and encoding four glycine codons in place of the parental HIHH codons.

Single $\mathrm{His}_{279}$ and $\operatorname{Arg}_{265}$ substitutions in the wecA gene were introduced by site-directed mutagenesis using QuikChange Site-Directed Mutagenesis Kit from Stratagene as recommended by the manufacturer. Plasmid pAA26 containing the $w e c A$ gene tagged with the FLAG sequence was used as a template in all reactions. Mutagenized plasmids were transformed into E. coli XL-1 Blue as recommended by the supplier. These experiments resulted in plasmids pAA51 $\left(w e c A_{\mathrm{H} 279 \mathrm{~s}}\right)$ and pAA52 $\left(w e c A_{\mathrm{R} 265 \mathrm{~K}}\right)$.

Transferase and binding assays. Both pAA26 encoding the parental wec $A_{\mathrm{FLAG}}$ gene and the other plasmids encoding the mutated wecA derivatives were transformed into strain MV501. Membranes were isolated as described by Osborn et al. (1972) after induction of the cultures with $0.02 \%$ arabinose for $3 \mathrm{~h}$. The reaction mixture for the transferase assay contained the membrane fraction ( $20 \mu \mathrm{g}$ total protein) and 96 pmol radiolabelled UDP-N-acetyl- $\left[{ }^{14} \mathrm{C}\right]$ glucosamine (225 mCi mmol ${ }^{-1}$; Amersham Pharmacia Biotech) in $250 \mu \mathrm{l}$ buffer $(5 \mathrm{mM}$ Tris-acetate, $\mathrm{pH} 8 \cdot 5,0 \cdot 1 \mathrm{mM}$ EDTA and $1 \mathrm{mM}$ $\left.\mathrm{MgCl}_{2}\right)$. After $30 \mathrm{~min}$ incubation at $37^{\circ} \mathrm{C}$, the lipid-associated material was extracted twice with $250 \mu \mathrm{l} 1$-butanol. The combined 1-butanol extracts were washed once with $500 \mu \mathrm{l}$ distilled water. The radioactive counts of the 1-butanol fraction were determined with a Beckman liquid scintillation counter. Radioactive counts were normalized and expressed as a percentage of parental $\mathrm{WecA}_{\mathrm{FLAG}}$ activity at $1 \mathrm{mM} \mathrm{MgCl}_{2}$ concentration (A. O. Amer \& M. A. Valvano, unpublished). An indirect assay that specifically measures the biosynthesis of $\mathrm{O} 7$ antigen by assaying the transfer of radiolabelled galactose into Und-P-P-GlcNAc acceptor in the presence of unlabelled UDP-GlcNAc was also used to determine WecA activity. The O7 polysaccharide repeating subunit consists of a backbone of GlcNAc, galactose, mannose and $\mathrm{N}$-acetylviosamine, and a side chain of rhamnose (L'Vov et al., 1984). Since WecA mediates the formation of Und-P-P-GlcNAc, the incorporation of radioactive galactose is strictly dependent on the presence of UDP-GlcNAc. This assay was carried out in a similar manner as described above except that the reaction mixture contained $0.5 \mu \mathrm{Ci}$ UDP- $\left[{ }^{3} \mathrm{H}\right]$ galactose (Amersham) and $0.08 \mathrm{mmol}$ UDP-GlcNAc.

Binding assays were performed as follows. Briefly, $40 \mu \mathrm{g}$ membranes from MV501 cells transformed with plasmids encoding the various wecA mutants were incubated with $45 \mathrm{ng}$ of the inhibitor tunicamycin for $10 \mathrm{~min}$ at room temperature. In previous experiments, we had determined that $45 \mathrm{ng}$ tunicamycin was the minimal amount of inhibitor required to inhibit the enzymic activity of $20 \mu \mathrm{g}$ membranes from MV501(pAA26) cells containing the parental WecA. The inhibitory activity of residual tunicamycin, which did not bind to the mutant proteins, was determined by adding $20 \mu \mathrm{g}$ membranes prepared from MV501(pAA26) in the presence of UDP-N-acetyl- $\left[{ }^{14} \mathrm{C}\right]$ glucosamine. Samples were incubated for $30 \mathrm{~min}$ at $37^{\circ} \mathrm{C}$ and processed with 1-butanol as described above for the transferase assay. Radioactive counts were expressed as a percentage of parental WecA activity assayed under the same conditions but in the absence of tunicamycin.

Immunoblot analysis. The same membrane preparations used for transferase and binding assays were subjected to Western
Blot analysis (Amer \& Valvano, 2000). Blots were developed using the FLAG M2 mAb as the primary antibody and horseradish peroxidase-linked sheep anti-mouse IgG (Amersham Pharmacia Biotechnology) as the secondary antibody. Detection by chemiluminescence was performed using the Chemiluminescence Blotting Substrate (Roche Diagnostics) as recommended by the manufacturer.

LPS analysis. LPS was extracted and analysed by SDS-PAGE followed by silver staining as previously described (Marolda et al., 1990).

Fractionation of membranes. The location of WecA and its mutated derivatives in the plasma membrane was verified by sucrose gradient fractionation of total bacterial membranes (Amer \& Valvano, 2000). Fractions were collected and assayed for NADH oxidase activity (Osborn et al., 1972) as a plasma membrane marker, and for the presence of outer membrane porins using SDS-PAGE to detect the outer membrane fractions (Amer \& Valvano, 2000). The presence of WecA in the fractions was examined by immunoblotting with the antiFLAG $\mathrm{mAb}$ as described above.

Amino acid sequence alignment. BLAST version 2 (Altschul et al., 1997) was used for homology searches in the database of non-redundant sequence. Amino acid sequence alignments of WecA homologues were performed using CLUSTAL W (Thompson et al., 1994). The transmembrane helices of WecA were predicted from the analysis of its amino acid sequence with TMHMM (Transmembrane Hidden Markov Model; Sonnhammer et al., 1998).

\section{RESULTS AND DISCUSSION}

\section{A topological model for WecA}

Analysis of the WecA amino acid sequence reveals alternating hydrophobic and hydrophilic segments that are consistent with a polytopic transmembrane protein structure. We used a hidden Markov model for predicting transmembrane helices within the WecA sequence. This model has recently been tested for high accuracy in a standard dataset of 83 membrane proteins whose topologies have been experimentally determined (Sonnhammer et al., 1998). The results suggested the presence of 11 transmembrane segments (Fig. 1a). The prediction also includes topological information on the orientation of the transmembrane helices, which is based on the fact that the positively charged residues arginine and lysine are mainly found in non-transmembrane parts of the protein on the cytoplasmic side (von Heijne, 1986, 1997). The predicted model for the topology of WecA was compared with that of the E. coli MraY protein (Fig. 1b). MraY belongs to the same family of proteins as WecA and its topology has been confirmed experimentally (Bouhss et al., 1999). Fig. 1 shows a good agreement between the predicted model for WecA and the established model for MraY, especially in regards to the topological location of non-transmembrane segments with high levels of amino acid sequence conservation, which are orientated towards the cytosolic compartment. We have recently discovered that regions in WecA corresponding to cytosolic segments II and III of MraY (Fig. 1) contain highly conserved aspartic acid residues (A. O. Amer \& M. A. Valvano, unpublished). 
(a)

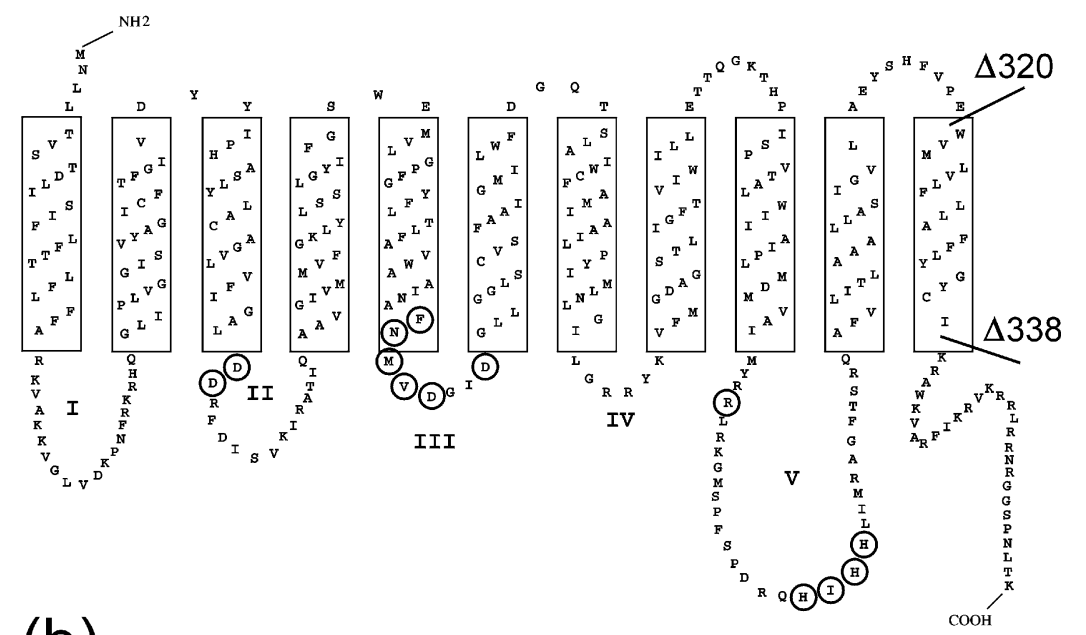

(b)

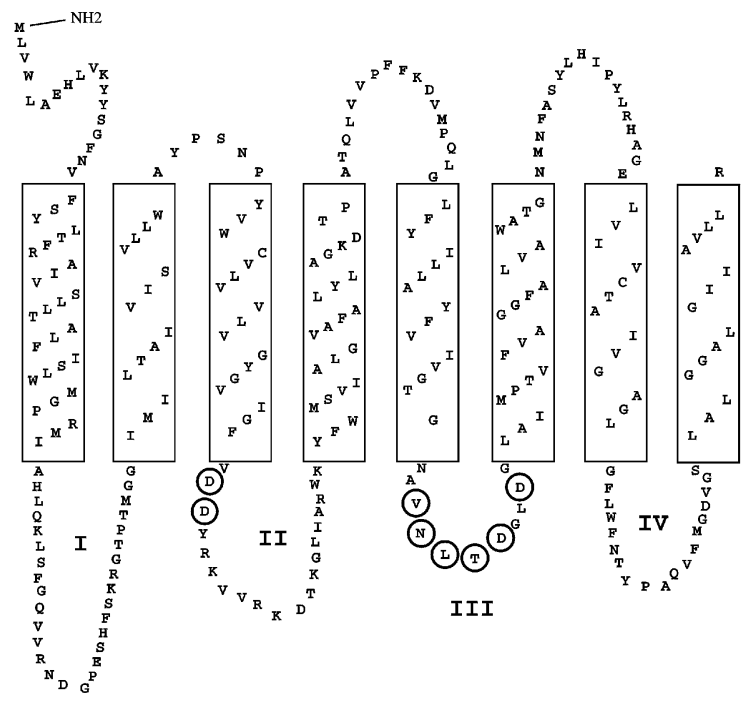

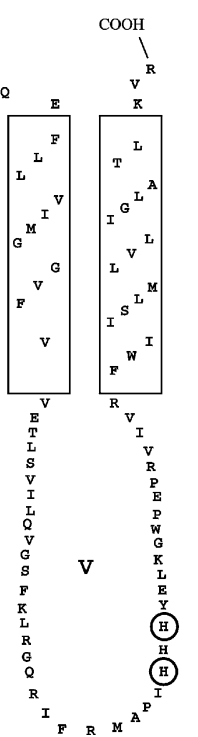

Fig. 1. Topological models of $E$. coli WecA and MraY transferases. (a) WecA model as predicted with TMHMM (Sonnhammer et al., 1998). The boundaries of deletions eliminating the C-terminal tail of WecA $(\Delta 338)$ and the last transmembrane segment $(\Delta 320)$ are indicated. (b) Topology of MraY as determined by Bouhss et al. (1999). In both models, the non-transmembrane segments that are exposed to the cytosol are indicated with roman numerals. Amino acids within circles denote those residues that are highly conserved within the WecA family $\left(D_{90}-D_{91}\right.$ in cytosolic segment II, $D_{156}$ and $D_{159}$ in cytosolic segment III, $R_{265}$ and $\mathrm{HIHH}_{279-282}$ in cytosolic segment $\mathrm{V}$ ). Amino acids $\mathrm{F}_{152}-\mathrm{N}_{153}-\mathrm{M}_{154}-\mathrm{V}_{155}$, which together with $D_{156}$ are characteristic of a Walker $B$ motif, are also indicated.
These residues are important for the biological function of WecA, and because of their conservation, they may also play a role in the function of the other bacterial and eukaryotic WecA homologues (A. O. Amer \& M. A. Valvano, unpublished).

In contrast to MraY, WecA has an additional transmembrane helix and a prominent C-terminal segment (Fig. 1). We have previously found that WecA does not require the last $27 \mathrm{C}$-terminal amino acids to remain functional (A. O. Amer \& M. A. Valvano, unpublished), and that a protein derivative containing a Cterminal FLAG epitope tag ( $\mathrm{WecA}_{\mathrm{FLAG}}$ ) has the same properties as wild-type WecA (Amer \& Valvano, 2000). The dispensable C-terminal region contains highly charged amino acids and has a net positive charge, suggesting a cytosolic location. This region is preceded by a predicted transmembrane helix containing 18 aa (Fig. 1a). To evaluate the possibility of using a deletionfusion approach with reporter proteins (Boyd et al., 1993; Prinz \& Beckwith, 1994) for providing experimental information on the topology of WecA, we investigated whether the most C-terminal transmembrane helix was required for WecA protein stability. Therefore, we constructed a deleted form of WecA $_{\text {FLAG }}$ lacking the C-terminal amino acids located downstream of a glutamic acid at position 320 (WecA ${ }_{\mathrm{E} 320}$; Fig. 1a). Since the FLAG epitope still remained fused to $\mathrm{WecA}_{\mathrm{E} 320}$, the expression of this protein was examined by Western blot with the FLAGspecific mAb M2. Fig. 2 shows that in contrast to the

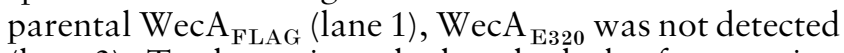
(lane 2). To determine whether the lack of expression was due to the absence of the missing transmembrane 


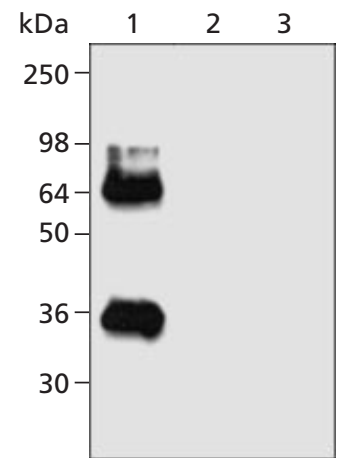

Fig. 2. Expression of $W_{e c} A_{E 320}$ and $W e c A_{\text {MalFTM. Western blot of }}$ a polyacrylamide gel containing whole-cell lysates of strain JT4000 transformed with the following plasmids: lane 1, pAA12 (encoding the FLAG-tagged wecA); lane 2, pAA10 (encoding

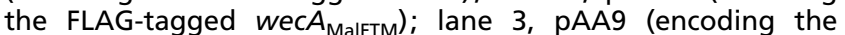
FLAG-tagged $\left.w e c A_{\mathrm{E320}}\right)$. The blot was developed using $\mathrm{mAb} M 2$ (anti-FLAG). The WecA monomer was identified at $\sim 36 \mathrm{kDa}$. Polypeptides of higher molecular mass can be detected under the conditions utilized for protein denaturation, which may represent oligomeric forms of WecA (Amer \& Valvano, 2000). The position of the following molecular mass standards is shown: myosin (250 kDa), bovine serum albumin $(98 \mathrm{kDa})$, glutamic acid dehydrogenase $(64 \mathrm{kDa})$, alcohol dehydrogenase (50 kDa), carbonic anhydrase (36 kDa) and myoglobin (30 kDa).

domain, we constructed another $\mathrm{WecA}_{\mathrm{FLAG}}$ derivative in which aa 321-339 were replaced by aa 476-506 of the MalF protein. These amino acids span the last transmembrane helix of MalF, which has similar hydrophobicity to that of WecA's last transmembrane helix (Boyd et al., 1987). The resulting derivative, $\mathrm{Wec}_{\text {MalFTM }}$, was also not detectable by Western blotting (Fig. 2, lane 3). The results of these experiments suggest that the last transmembrane helix of WecA is essential for the stability of the protein, perhaps by interacting with other transmembrane segments or by playing a role in targeting the protein to the plasma membrane. Furthermore, we have previously demonstrated that although WecA can tolerate a substantial deletion of N-terminal amino acids, resulting in the loss of at least three predicted transmembrane helices and still insert in the plasma membrane, the protein is no longer functional (Amer \& Valvano, 2000). Thus, a practical outcome of these results is that they precluded us from using a sequential deletion-fusion strategy to experimentally map the topology of WecA, as it has been performed with MraY (Bouhss et al., 1999).

\section{Conserved residues in the predicted large cytosolic loop of WecA that are involved in the recognition of UDP-GICNAC}

The topological model of WecA predicts a relatively large (32 aa) non-transmembrane segment exposed to the cytosolic face of the plasma membrane (Fig. 1a). This segment corresponds to a similar region also found in the MraY protein (Fig. 1b). Sequence comparisons with additional members of this family indicate that this region is particularly conserved in the bacterial homologues of WecA (Anderson et al., 2000). Alignment of the amino acid sequences of WecA homologues shows that $\operatorname{Arg}_{265}$ and $\mathrm{His}_{279}$ (the numbers refer to the position of these amino acids in the E. coli $\mathrm{K}-12$ WecA protein) are highly conserved (Fig. 3). Furthermore, in WecA homologues from E. coli, Salmonella enterica serovar Typhimurium and Deinococcus radiodurans, and in the TagO protein from Bacillus subtilis, the highly conserved $\mathrm{His}_{279}$ is part of a less conserved short sequence motif HIHH (His-Ile-His-His; Fig. 3). This motif bears resemblance to the HIGH (His-Ile-Gly-His) motif identified in class I aminoacyl-tRNA synthetases and also in the superfamily of related nucleotidyltransferases (Bork et al., 1995; Sekine et al., 2001; Venkatachalam et al., 1999). In these enzymes, the HIGH motif constitutes a portion of the three-dimensional structure of the ATPbinding site and is implicated in nucleotide binding as well as in $\alpha / \beta$ phosphodiesterase activity (Sekine et al., 2001; Venkatachalam et al., 1999). Given that the HIGH motif has a role in nucleotide phosphate binding, it is conceivable that a similar structural motif in WecA may be involved in binding of the nucleotide substrate UDP$\mathrm{N}$-acetylhexosamine and the release of UMP, a step that would also necessitate phosphodiesterase activity.

To ascertain the role of the HIHH motif in WecA function, the four residues were substituted by glycine, resulting in the $\mathrm{WecA}_{\mathrm{HIHH} / \mathrm{GGGG}}$ mutant (pAA33). Glycine was preferred for the replacements to avoid the use of hydrophobic residues like alanine for amino acid replacements, which could dramatically alter the topology of a non-transmembrane region. Topological predictions on the mutant protein using TMHMM (Transmembrane Hidden Markov Model) showed results identical to wild-type WecA. We also replaced the highly conserved $\mathrm{His}_{279}$ residue individually with serine, resulting in $\mathrm{WecA}_{\mathrm{H} 279 \mathrm{~S}}$. Serine was used for the single replacement because it is considered to be a conservative substitution for histidine (Bordo \& Argos, 1991). Plasmids harbouring $w e c A_{\mathrm{FLAG}}(\mathrm{pAA} 26)$, as well as the mutated $w e c A_{\mathrm{HIHH} / \mathrm{GGGG}}(\mathrm{pAA} 33)$ and $w e c A_{\mathrm{H} 279 \mathrm{~S}}$ (pAA51) genes were transformed into E. coli MV501. This strain, a derivative of E. coli VW187 containing a wecA:: Tn10 insertion, is unable to form O7-specific LPS (Alexander \& Valvano, 1994). Expression of WecA $A_{\text {FLAG }}$, WecA $A_{\text {HIHH/GGGG }}$ and $\mathrm{WecA}_{\mathrm{H} 279 \mathrm{~S}}$ was monitored under induction with $0.02 \%$ arabinose. Fig. 4a shows that, in contrast to parental $\mathrm{WecA}_{\mathrm{FLAG}}$ (lane 1), $\mathrm{WecA}_{\mathrm{HIHH} / \mathrm{GGGG}}$ was unable to restore the formation of the characteristic O7 LPS ladder in MV501 (lane 3). Although $\mathrm{WecA}_{\mathrm{H} 279 \mathrm{~S}}$ also did not restore the characteristic O7 polysaccharide ladder, a reduced level of activity was present as indicated by the formation of LPS containing a single $\mathrm{O} 7$ subunit (Fig. 4a, lane 4). To rule out the possibility that the amino acid replacements could have affected either protein stability or targeting to the plasma membrane, the levels of expression of the two constructs were examined by Western blot using the M2 FLAG-specific mAb. WecA $\mathrm{HHHH}_{\text {/GGGG }}$ and $\mathrm{Wec}_{\mathrm{H} 279 \mathrm{~S}}$ proteins showed comparable levels of ex- 


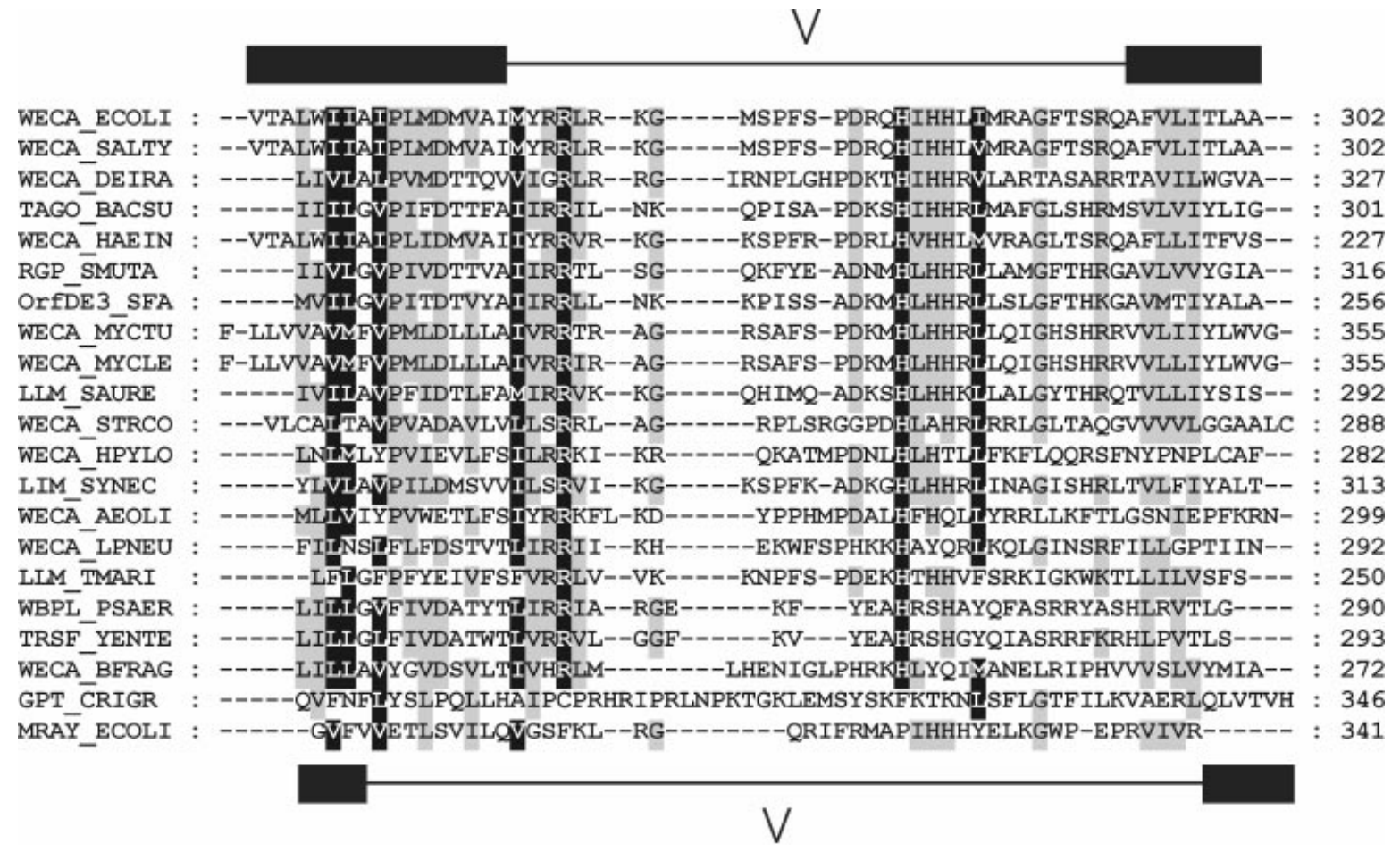

Fig. 3. Alignment of bacterial WecA homologues. WecA homologues were identified from a BLAST search using the $E$. coli WecA protein as a query. Alignments were determined with the program CLUSTAL $W$. The figure shows a partial alignment of the region spanning the cytosolic segment $V$ and part of the two neighbouring transmembrane domains (indicated with black bars). The boundaries of the transmembrane domains were defined according to the predicted model for WecA (top) and the established model for MraY (bottom). Amino acids presented with a black background denote highly conserved residues across the proteins examined. The sequences from the following organisms were compared (accession numbers are shown in parentheses): WECA_ECOLI, E. coli (P24235); WECA_SALTY, Salmonella typhimurium strain LT2 (AAF33469.1); WECA_DEIRA, Deinococcus radiodurans (B75380); TAGO_BACSU, Bacillus subtilis (B69721); WECA_HAEIN, Haemophilus influenzae (P45341); RGP_SMUTA, Streptococcus mutans (BAA82114.1); OrfDE3_SFA, S. faecalis (AAC35916.1); WECA_MYCTU, Mycobacterium tuberculosis (Q10606); WECA_MYCLE, Mycobacterium leprae (P45830); LLM_SAURE, Staphylococcus aureus (A55856); WECA_STRCO, Streptomyces coelicolor (T36607); WECA_HPYLO, Helicobacter pylori strain $J 99$ (B71801); LIM_SYNEC, Synechocystis sp. (S74927); WECA_AEOLI, Aquifex aeolicus (H70346); WECA_LPNEU, Legionella pneumophila (CAB65202.1); LLM_TMARI, Thermotoga maritima (H72238); WBPL_PSAER, Pseudomonas aeruginosa serotype O5 (AAC43404.1); TRSF_YENTE, Yersinia enterocolitica (S51265); WECA_BFRAG, Bacteroides fragilis (AAD40724.1); GPT_CRIGR, Cricetulus griseus (P24140); and MRAY_ECOLI, E. coli (P15876).

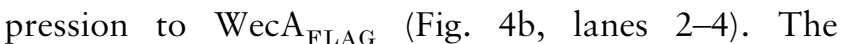
localization of the mutant proteins in the plasma membrane was examined by sucrose gradient centrifugation. These experiments revealed that both parental and mutant proteins were localized in the fractions containing cytoplasmic membrane components. Indeed, these fractions demonstrated a high level of NADH oxidase activity and they lacked outer membrane porins (data not shown). We concluded that the inability of $\mathrm{WecA}_{\mathrm{HHHH} / \mathrm{GGGG}}$ and $\mathrm{WecA}_{\mathrm{H} 279 \mathrm{~S}}$ to complement $\mathrm{O} 7$ LPS synthesis in vivo was due to a defect in the enzymic activity of WecA rather than a topological artefact introduced by the amino acid replacements.

The enzymic activity of WecA and the mutant proteins was investigated in more detail using two in vitro assays for determining the transfer of UDP-GlcNAc to Und-P. One assay determined the direct incorporation of radiolabelled $\left[{ }^{14} \mathrm{C}\right] \mathrm{GlcNAc}$ (provided in the reaction as UDP-GlcNAc) into a butanol-extractable fraction, which contains Und-P-P-GlcNAc intermediates. Previous studies in our laboratory showed that WecA initiates the synthesis of the $\mathrm{O} 7$ oligosaccharide subunit by forming Und-P-P-linked GlcNAc. This glycolipid then serves as the acceptor for the galactose, the subsequent sugar of the O7 subunit (Alexander \& Valvano, 1994; Marolda et al., 1999). Therefore, in the second assay we determined the UDP-GlcNAc-dependent incorporation of radiolabelled $\left[{ }^{3} \mathrm{H}\right] \mathrm{Gal}$ (provided as UDP-Gal) into the butanol-extractable fraction. This reaction served as a control to rule out the possibility that GlcNAc may have also been added to Und-P-P-Nacetylmuramoyl-pentapeptide, which serves as a peptidoglycan biosynthesis intermediate, by the endogenous activity of the MraY enzyme that is present in the crude membrane extract. Membrane extracts containing endogenous polyprenyl phosphate acceptor were prepared from MV501 transformed with the various plasmids encoding $w e c A_{\mathrm{FLAG}}$ or its mutated derivatives. The results of the direct transferase assay demonstrated 


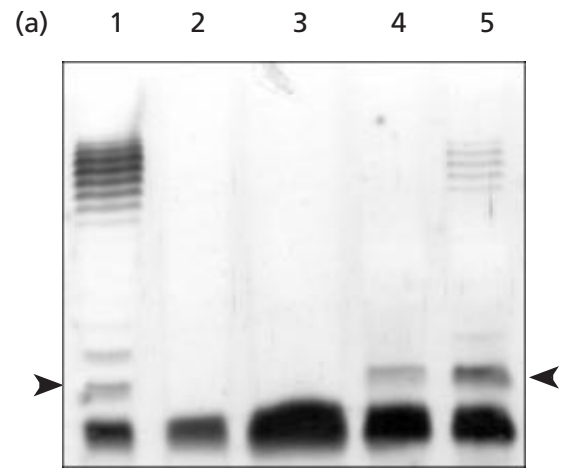

(b)

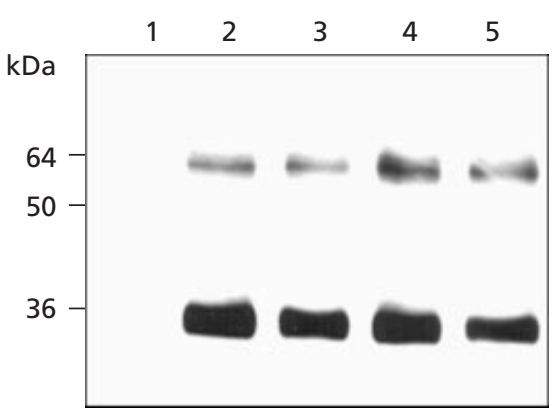

Fig. 4. (a) $O 7$ LPS synthesis in strain MV501 containing the parental $w e c A_{\mathrm{FLAG}}$ and mutated wecA genes. LPS samples were obtained from $E$. coli MV501 transformed with the following plasmids: lane 1, pAA26 (encoding wecA $A_{\mathrm{FLAG}}$ ); lane 2, pBAD24; lane 3, pAA33 (encoding $w_{e c} A_{\mathrm{HIH} / \mathrm{GGGG}}$ ); lane 4, pAA51 (encoding $w e c A_{\mathrm{H} 279 \mathrm{~S}}$ ); and lane 5, pAA52 (encoding $w e c A_{\mathrm{R} 265 \mathrm{~K}}$ ). LPS was separated by SDS-PAGE and silver stained. Arrows indicate the lipid A core plus one 07 subunit. (b) Expression of mutant WecA protein derivatives. Bacterial cell membranes were prepared from strain MV501 transformed with the following plasmids: pBAD24 (lane 1), pAA26 (lane 2), pAA33 (lane 3), pAA51 (lane 4) and pAA52 (lane 5). The positions of the molecular mass standards are shown: glutamic acid dehydrogenase (64 kDa), alcohol dehydrogenase (50 kDa) and carbonic anhydrase (36 kDa) were used.

that membranes from cells expressing $\mathrm{Wec}_{\mathrm{HHHH} / \mathrm{GGGG}}$ and $\mathrm{WecA}_{\mathrm{H} 279 \mathrm{~S}}$ had virtually no enzymic activity, since the levels of activity were lower than those observed with membranes from cells carrying the control plasmid pBAD24 (Table 3). The low background in control membranes demonstrated that the incorporation of radioactive sugar into the non-polar butanol fraction was only due to WecA enzyme activity. The indirect assay using radioactive UDP-Gal gave very similar results (data not shown), confirming that GlcNAc 1phosphate was directly incorporated to Und-P instead of a peptidoglycan biosynthesis intermediate.

We have previously shown that when WecA and the Oantigen translocase Wzx are present a terminal GlcNAc is attached to the E. coli lipid A core, which can be detected using digoxigenin-labelled wheat germ agglutinin (Feldman et al., 1999). This assay was used as an independent confirmation of the functionality of the WecA mutants. In this case, E. coli strain CLM20 was utilized, as this strain lacks the genes necessary for the transfer and the translocation of the sugars involved in the biosynthesis of the O-specific LPS (Feldman et al., 1999). CLM20 was co-transformed with pAA33 or pAA51 (expressing $\mathrm{WecA}_{\mathrm{HHHH} / \mathrm{GGGG}}$ and $\mathrm{WecA}_{\mathrm{H} 279 \mathrm{~S}}$, respectively), and pMF21 (encoding the O-antigen translocase Wzx). Fig. 5a, lane 3, shows that no GlcNAc was detected in the LPS core obtained from cells expressing $\mathrm{Wec}_{\mathrm{HHHH} / \mathrm{GGGG}}$. In contrast, a small amount of terminal GlcNAc was detected in the LPS core of cells expressing $\mathrm{WecA}_{\mathrm{H} 279 \mathrm{~S}}$ as compared to the

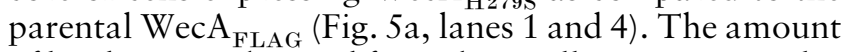
of lipid A core obtained from these cells was very similar (Fig. 5b), thus ruling out the possibility that these differences are due to a loading artefact. Taken together, these results demonstrate that the failure to form O7 polysaccharide chain in vivo correlates with absent or reduced in vitro transfer activity of $\mathrm{Wec}_{\mathrm{HHHH} / \mathrm{GGGG}}$ and $\mathrm{WecA}_{\mathrm{H} 279 \mathrm{~S}}$, respectively.

The previous experiments did not distinguish whether the mutations have an effect on the ability of WecA to bind the nucleotide-sugar substrate or on the mechanism of transfer of GlcNAc 1-phosphate to Und-P. To differentiate between these possibilities, we used a binding assay involving the use of the UDP-GlcNAc analogue tunicamycin, which inhibits the activity of the enzyme (Dal Nogare \& Lehrman, 1988). We reasoned that mutations in a putative binding region that would prevent the interaction between WecA and UDPGlcNAc would also prevent the binding of tunicamycin. Membrane preparations containing the mutated WecA forms were treated with minimal amounts of tunicamycin and the presence of residual inhibitor was determined by assaying the activity of the parental $\mathrm{WecA}_{\mathrm{FLAG}}$. Table 3 shows that membranes containing $\mathrm{WecA}_{\mathrm{HHHH} / \mathrm{GGGG}}$ and $\mathrm{Wec}_{\mathrm{H} 279 \mathrm{~S}}$ displayed a markedly reduced tunicamycin binding as compared to membranes containing $\mathrm{Wec}_{\mathrm{FLAG}}$. We conclude from these experiments that the amino acid replacements affected the ability of WecA to bind UDP-GlcNAc, and probably identify critical residues in the protein that may be involved in the recognition of the nucleotide-sugar substrate. Furthermore, the relative conservation of the $\mathrm{HIHH}$ motif and the high conservation of $\mathrm{His}_{279}$ in the other members of the WecA protein family support the idea that these residues play a functional role. The conserved $\mathrm{His}_{279}$ is also the first residue of the $\mathrm{HIHH}$ motif. Interestingly, mutagenesis studies in enzymes with the HIGH motif have shown that the first His of the motif is critical for enzymic activity (Venkatachalam et al., 1999).

Structural analysis of class I tRNA synthetases has revealed another short sequence motif, KMSK (LysMet-Ser-Lys), that is involved in the three-dimensional architecture of the ATP-binding site (Sekine et al., 2001). It was suggested that the second lysine of the KMSK motif plays a key role in catalysis, as it interacts with the $\alpha$-phosphate of the ATP molecule by forming a salt bridge with the phosphate group of the adenylate (Sekine et al., 2001). We suggest that the positive charge at 
Table 3. Comparative transfer and binding activities of $\mathrm{WeCA}_{\mathrm{FLAG}}$ and its mutated derivatives

\begin{tabular}{|llcc|}
\hline Membrane extract* & WecA protein & Transfer assay $\dagger$ & Binding assay $\neq$ \\
\hline pBAD24 & None & $10 \cdot 9 \pm 6 \cdot 3$ & $12 \cdot 8 \pm 0 \cdot 9$ \\
pAA26 & WecA $_{\mathrm{FLAG}}$ & 100 & 100 \\
pAA33 & WecA $_{\mathrm{H} \text { HH } / \mathrm{GGGG}}$ & $7 \cdot 5 \pm 2 \cdot 5$ & $10 \cdot 2 \pm 1 \cdot 1$ \\
pAA51 & WecA $_{\mathrm{H} 279 \mathrm{~S}}$ & $7 \cdot 9 \pm 2 \cdot 7$ & $15 \cdot 1 \pm 2 \cdot 4$ \\
pAA52 & WecA $_{\mathrm{R} 265 \mathrm{~K}}$ & $44 \cdot 5 \pm 18 \cdot 4$ & $48 \cdot 3 \pm 11 \cdot 6$ \\
\hline
\end{tabular}

* Membrane extracts were prepared from E. coli MV510 cells transformed with the indicated plasmids. † Incorporation of radioactive GlcNAc into a butanol-extractable fraction as described in Methods. The results, expressed as a percentage of the parental $\mathrm{WecA}_{\mathrm{FLAG}}$ activity at $1 \mathrm{mM} \mathrm{MgCl}_{2}$ using normalized counts, represent the mean \pm SD of three independent experiments.

$\ddagger$ Incorporation of radioactive GlcNAc into a butanol-extractable fraction in competition with residual tunicamycin as described in Methods. The results, expressed as a percentage of the parental WecA $\mathrm{FLAG}_{\mathrm{F}}$ activity at $1 \mathrm{mM} \mathrm{MgCl}$ using normalized counts, represent the mean $\pm \mathrm{SD}$ of three independent experiments.

(a)

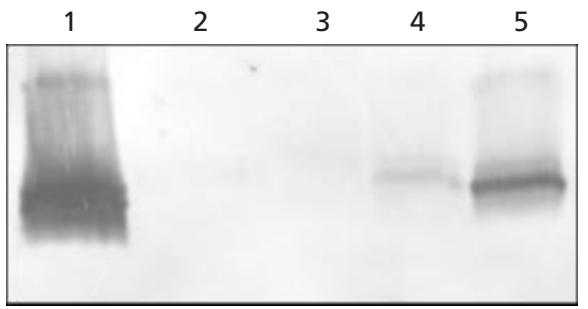

(b)

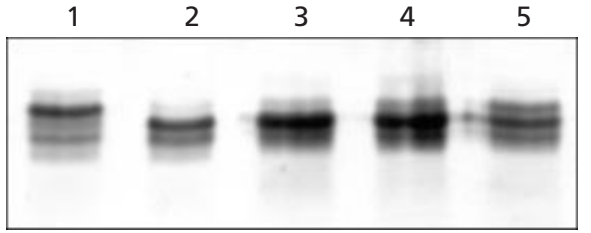

Fig. 5. (a) Lectin-Western blot. LPS samples were extracted from $E$. coli strain CLM20(pMF21) transformed with the following plasmids: pAA26, encoding $w_{\text {ec }} A_{\text {FLAG }}$ (lane 1); pBAD24 (lane 2); pAA33, encoding $w_{\text {ecc }} A_{\text {HHH/GGGG }}$ (lane 3); pAA51, encoding $w{ }^{2} A_{H 279 s}$ (lane 4); and pAA52, encoding $w_{e} A_{R 265 K}$ (lane 5). Western blotting was performed using digoxigenin-labelled wheat-germ agglutinin and horseradish peroxidase labelled anti-digoxigenin antibodies (Feldman et al., 1999). (b) Silver-stained gel of the same samples used in (a).

position 265 could be involved in binding the phosphates of UDP-GlcNAc, similar to the role of the second lysine in KMSK. The proposed function for $\operatorname{Arg}_{265}$ is supported by its high level of conservation among WecA homologues, within a region containing a cluster of additional positively charged residues (Fig. 3). A conservative replacement of $\operatorname{Arg}_{265}$ with lysine was made to determine whether this residue has any role in WecA function. The mutant protein, $\mathrm{Wec}_{\mathrm{R} 265 \mathrm{~K}}$, was expressed at comparable levels with respect to $\mathrm{WecA}_{\mathrm{FLAG}}$ and was localized in the plasma membrane (Fig. $4 \mathrm{~b}$, lane 5 and data not shown). $\mathrm{WecA}_{\mathrm{R} 265 \mathrm{~K}}$ showed a partial com- plementation of $\mathrm{O} 7$ polysaccharide biosynthesis in vivo (Fig. 4a, lane 5) which correlated with a $44 \%$ and $48 \%$ reduction in the levels of transferase and binding activities in vitro, respectively (Table 3 ). These results cannot be explained by a topological defect, since the conserved replacement of $\operatorname{Arg}_{265}$ would not affect the overall positive charge of the non-transmembrane segment, suggesting that the decreased functionality of the mutant WecA may be due to a defect in substrate binding. We speculate that the replacement of arginine with lysine decreases the affinity of the protein for UDPGlcNAc. However, further experiments involving purified protein are required to confirm this conclusion.

\section{Concluding remarks}

In this study, we have compared the predicted topology of WecA with that of the MraY protein, whose topology has been determined experimentally (Bouhss et al., 1999). Both proteins contain a large cytosolic-exposed non-transmembrane region, which contains conserved amino acid residues, especially $\mathrm{Arg}_{265}$ and $\mathrm{His}_{279}$. These and additional residues resemble key amino acids found in nucleotidyl transferases. The analysis of WecA mutant proteins containing amino acid replacements of these key residues strongly suggests their involvement in the recognition of UDP-GlcNAc. Since WecA can also complement the biosynthesis of $\mathrm{O}$ antigens containing $\mathrm{N}$-acetylgalactosamine, other investigators have suggested that WecA has a loose substrate specificity, which involves the recognition of UDP- $N$-acetylgalactosamine in addition to UDP-GlcNAc (Amor \& Whitfield, 1997; Zhang et al., 1997). Future studies involving purified WecA, currently under way in our laboratory, are needed to clarify its substrate specificity. At any rate, the amino acids identified in this study are also conserved in other WecA homologues such as Yersinia enterocolitica $\mathrm{WbcO}$ and Pseudomonas aeruginosa $\mathrm{WbpL}$, which have predicted specificity for UDP- 
$\mathrm{N}$-acetylfucosamine (Skurnik, 1999). Therefore, it is possible that these amino acid residues may have a general role in the function of these proteins, perhaps by contributing to the architecture of a common fold required for the recognition of UDP- $\mathrm{N}$-acetylhexosamines.

\section{ACKNOWLEDGEMENTS}

This study was supported by grant MT-10206 from the Canadian Institutes of Health Research to M.A.V. A.O.A. was supported by an Ontario Graduate Scholarship Award.

\section{REFERENCES}

Alexander, D. C. \& Valvano, M. A. (1994). Role of $r f e$ gene in the biosynthesis of the Escherichia coli O7-specific lipopolysaccharide and other $\mathrm{O}$-specific polysaccharides containing $\mathrm{N}$-acetylglucosamine. J Bacteriol 176, 7079-7084.

Altschul, S. F., Madden, T. L., Schaffer, A. A., Zhang, J., Zhang, Z., Miller, W. \& Lipman, D. J. (1997). Gapped BLAST and PSI-BLAST : a new generation of protein database search programs. Nucleic Acids Res 25, 3389-3402.

Amer, A. O. \& Valvano, M. A. (2000). The N-terminal region of the Escherichia coli WecA (Rfe) protein, containing three predicted transmembrane helices, is required for function but not for membrane insertion. J Bacteriol 182, 498-503.

Amor, P. A. \& Whitfield, C. (1997). Molecular and functional analysis of genes required for expression of group IB $\mathrm{K}$ antigens in Escherichia coli: characterization of the his region containing gene clusters for multiple cell-surface polysaccharides. Mol Microbiol 26, 145-161.

Anderson, M. S., Eveland, S. S. \& Price, N. P. (2000). Conserved cytoplasmic motifs that distinguish sub-groups of the polyprenol phosphate: $\mathrm{N}$-acetylhexosamine-1-phosphate transferase family. FEMS Microbiol Lett 191, 169-175.

Bordo, D. \& Argos, P. (1991). Suggestions for 'safe' residue substitutions in site-directed mutagenesis. J Mol Biol 217, 721-729.

Bork, P., Holm, L., Koonin, E. V. \& Sander, C. (1995). The cytidylyltransferase superfamily: identification of the nucleotidebinding site and fold prediction. Proteins 22, 259-266.

Bouhss, A., Mengin-Lecreulx, D., Le Beller, D. \& Van Heijenoort, J. (1999). Topological analysis of the MraY protein catalysing the first membrane step of peptidoglycan synthesis. Mol Microbiol 34, 576-585.

Boyd, D., Manoil, C. \& Beckwith, J. (1987). Determinants of membrane protein topology. Proc Natl Acad Sci USA 84, 8525-8529.

Boyd, D., Traxler, B. \& Beckwith, J. (1993). Analysis of the topology of a membrane protein by using a minimum number of alkaline phosphatase fusions. J Bacteriol 175, 553-556.

Cohen, S. N., Chang, A. C. \& Hsu, L. (1972). Nonchromosomal antibiotic resistance in bacteria: genetic transformation of Escherichia coli by R-factor DNA. Proc Natl Acad Sci US A 69, 2110-2114.

Dal Nogare, A. R. \& Lehrman, M. A. (1988). Conserved sequences in enzymes of the UDP-GlcNAc/MurNAc family are essential in hamster UDP-GlcNAc: dolichol-P GlcNAc-1-P transferase. Glycobiology 8, 625-632.

Dan, N., Middleton, R. B. \& Lehrman, M. A. (1996). Hamster UDP$\mathrm{N}$ - acetylglucosamine: dolichol-P $\mathrm{N}$-acetylglucosamine-1-P trans- ferase has multiple transmembrane spans and a critical cytosolic loop. J Biol Chem 271, 30717-30724.

Dower, W. J., Miller, J. F. \& Ragsdale, C. W. (1988). High efficiency transformation of $E$. coli by high voltage electroporation. Nucleic Acids Res 16, 6127-6145.

Feldman, M. F., Marolda, C. L., Monteiro, M. A., Perry, M. B., Parodi, A. J. \& Valvano, M. A. (1999). The activity of a putative polyisoprenol-linked sugar translocase (Wzx) involved in Escherichia coli $\mathrm{O}$ antigen assembly is independent of the chemical structure of the O repeat. J Biol Chem 274, 35129-35138.

Guzman, L. M., Belin, D., Carson, M. J. \& Beckwith, J. (1995). Tight regulation, modulation, and high-level expression by vectors containing the arabinose $\mathrm{P}_{\mathrm{BAD}}$ promoter. J Bacteriol 177, 4121-4130.

von Heijne, G. (1986). The distribution of positively charged residues in bacterial inner membrane proteins correlates with the trans-membrane topology. EMBO J 5, 3021-3027.

von Heijne, G. (1997). Getting greasy: how transmembrane polypeptide segments integrate into the lipid bilayer. Mol Microbiol 24, 249-253.

Lehrman, M. A. (1994). A family of UDP-GlcNAc/MurNAc: polyisoprenol-P GlcNAc/MurNAc-1-P transferases. Glycobiology 4, 768-771.

L'Vov, V., Shashkov, A. S., Dmitriev, B. A., Kochetkov, N. K., Jann, B. \& Jann, K. (1984). Structural studies of the O-specific side chain of the lipopolysaccharide from Escherichia coli O:7. Carbohydr Res 126, 249-259.

Marolda, C. L., Welsh, J., Dafoe, L. \& Valvano, M. A. (1990). Genetic analysis of the O7-polysaccharide biosynthesis region from the Escherichia coli O7-K1 strain VW187. J Bacteriol 172, 3590-3599.

Marolda, C. L., Feldman, M. F. \& Valvano, M. A. (1999). Genetic organization of the O7-specific lipopolysaccharide biosynthesis cluster of Escherichia coli VW187 (O7:K1). Microbiology 145, 2485-2495.

Osborn, M. J., Gander, J. E., Parisi, E. \& Carson, J. (1972). Mechanism of assembly of the outer membrane of Salmonella typhimurium: isolation and characterization of cytoplasmic and outer membrane. J Biol Chem 247, 3962-3972.

Prinz, W. A. \& Beckwith, J. (1994). Gene fusion analysis of membrane protein topology: a direct comparison of alkaline phosphatase and beta-lactamase fusions. J Bacteriol 176, 6410-6413.

Rick, P. D. \& Silver, R. P. (1996). Enterobacterial common antigen and capsular polysaccharides. In Escherichia coli and Salmonella: Cellular and Molecular Biology, pp. 104-122. Edited by F. C. Neidhardt and others. Washington, DC: American Society for Microbiology.

Sekine, S., Shimada, A., Nureki, O., Cavarelli, J., Moras, D., Vassylyev, D. \& Yokoyama, S. (2001). Crucial role of the HIGHloop lysine for the catalytic activity of arginyl-tRNA synthetase. J Biol Chem 276, 3723-3726.

Skurnik, M. (1999). Molecular genetics of Yersinia lipopolysaccharide. In Genetics of Bacterial Polysaccharides, pp. 23-51. Edited by J. Goldberg. Boca Raton, FL: CRC Press.

Sonnhammer, E. L. L., von Heijne, G. \& Krogh, A. (1998). A hidden Markov model for predicting transmembrane helices in protein sequences. In Proceedings of Sixth International Conference on Intelligent Systems for Molecular Biology, pp. 175-182. Edited by J. Glasgow and others. Menlo Park, California: American Association for Artificial Intelligence.

Thompson, J. D., Higgins, D. G. \& Gibson, T. J. (1994). CluSTAL W : 
improving the sensitivity of progressive multiple sequence alignment through sequence weighting, position specific gap penalties and weight matrix choice. Nucleic Acids Res 22, 4673-4680.

Venkatachalam, K. V., Fuda, H., Koonin, E. V. \& Strott, C. A. (1999). Site-selected mutagenesis of a conserved nucleotide binding HXGH motif located in the ATP sulfurylase domain of human bifunctional 3'-phosphoadinosine 5'-phosphosulfate synthase. J Biol Chem 274, 2601-2604.
Zhang, L., Radziejewska-Lebrecht, J., Krajewska-Pietrasik, D., Toivanen, P. \& Skurnik, M. (1997). Molecular and chemical characterization of the lipopolysaccharide $\mathrm{O}$-antigen and its role in virulence of Yersinia enterocolitica serotype O:8. Mol Microbiol 23, 63-76.

Received 1 June 2001; revised 20 June 2001; accepted 9 July 2001. 